\title{
In-situ Deformation of Various Micro/Nanoscaled Samples in the Transmission Electron Microscope: Experimental Results and Pitfalls
}

\author{
Rohit Sarkar ${ }^{1}$, Jagannathan Rajagopalan ${ }^{1}$, Christian Ebner ${ }^{2}$ and Christian Rentenberger ${ }^{2}$ \\ 1. School for Engineering of Matter Transport and Energy, Arizona State University, Tempe, USA. \\ 2. Physics of Nanostructured Materials, Faculty of Physics, University of Vienna, Vienna, Austria.
}

Properties including mechanical properties of micro/nanoscaled devices are of great technological importance and scientific interest. In order to obtain simultaneously both mechanical information and structural changes on atomic and nano scale, in-situ experiments in the transmission electron microscope (TEM) are supposed to be most appropriate. In the following paper we present (i) experimental TEM results obtained by in-situ tensile tests of nanocrystalline metals and metallic glasses and (ii) point to possible pitfalls leading to artifacts.

Freestanding microscaled amorphous TiAl samples as well as nanocrystalline Al and Au thin films were produced on MEMS devices using DC magnetron sputtering and microfabrication techniques (Figure 1). The MEMS based tensile devices have built-in force and displacement sensing gauges (cf. 1-3 in Fig. 1) to measure macroscopic stress and strain during in-situ tensile deformation. A Philips straining holder was used to deform various films in a Philips CM200 TEM equipped with a Gatan Orius ${ }^{\text {TM }}$ CCD camera. Bright-field and selected area electron diffraction (SAED) images were acquired at different deformation states of the films. In addition, time series or movies were taken to gain information on dislocation activity and film relaxation.

In the case of amorphous TiAl atomic-level elastic strains were measured during in-situ deformation by quantification of the first diffraction halo in SAED patterns. The full evaluation of the distortion of the diffraction ring giving the 2D strain tensor was carried out automatically by a plug-in written for the Digital Micrograph $^{\mathrm{TM}}$ platform [1]. Figure 2a shows a characteristic SAED pattern and the corresponding parameters. From the deformation-induced peak shift with respect to a reference image the atomic-level strain as function of the azimuthal angle (cf. Fig. 2b) can be calculated. The linear fit of the principal strains as function of stress (cf. Fig. 2c) yields the Young's modulus and the Poisson's ratio. The comparison with the principal strain measured at the gauges reveals the presence of anelasticity.

In nanocrystalline Al thin films in-situ straining experiments provide insight into the deformation mechanism and motion of dislocations. Figure 3a shows a snapshot of a movie taken at an accelerating voltage of $120 \mathrm{kV}$. Dislocations that were active $7 \mathrm{sec}$ after a deformation pulse at a stress of $225 \mathrm{MPa}$ are indicated by red arrows. By comparing the stress-strain curves and relaxation at different accelerating voltages (cf. Fig. 3b) it is concluded that the TEM electron beam causes enhanced dislocation mobility. In addition to relaxation, e-beam exposure causes also local necking of the film [2]. Therefore, imaging conditions should be chosen carefully to avoid beam-induced artifacts leading to misinterpretation [3].

References:

[1] C. Ebner et al, Ultramicroscopy 165 (2016) p. 51.

[2] R. Sarkar, C. Rentenberger, J. Rajagopalan, Sci. Rep. 5 (2015) p. 16345.

[3] C. E. and C. R. acknowledge financial support by the Austrian Science Fund FWF: [I1309]. R. S. 
and J. R. acknowledge funding from the NSF grants CMMI 1400505 and DMR 1454109.

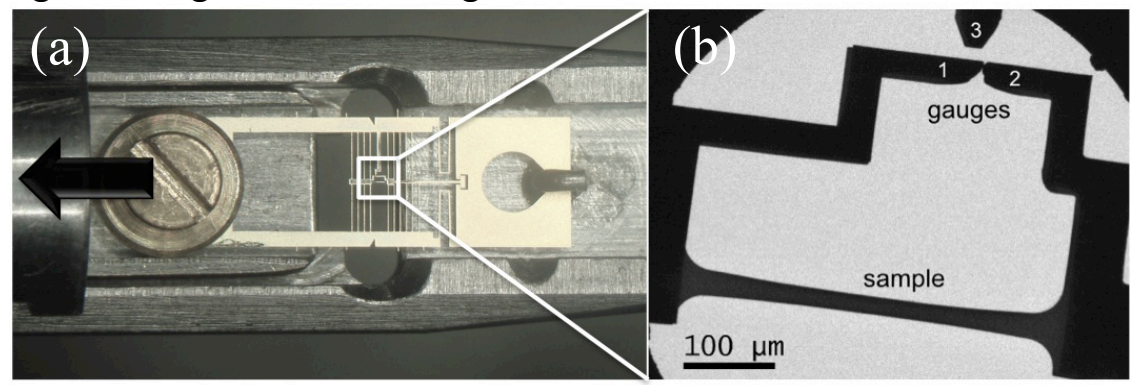

Figure 1. (a) MEMS device mounted in a straining holder allow uniaxial tensile deformation of a freestanding nanocrystalline or amorphous thin film. (b) Enlarged view of the central part shows sample, strain (1-2) and force (1-3) sensing gauges.
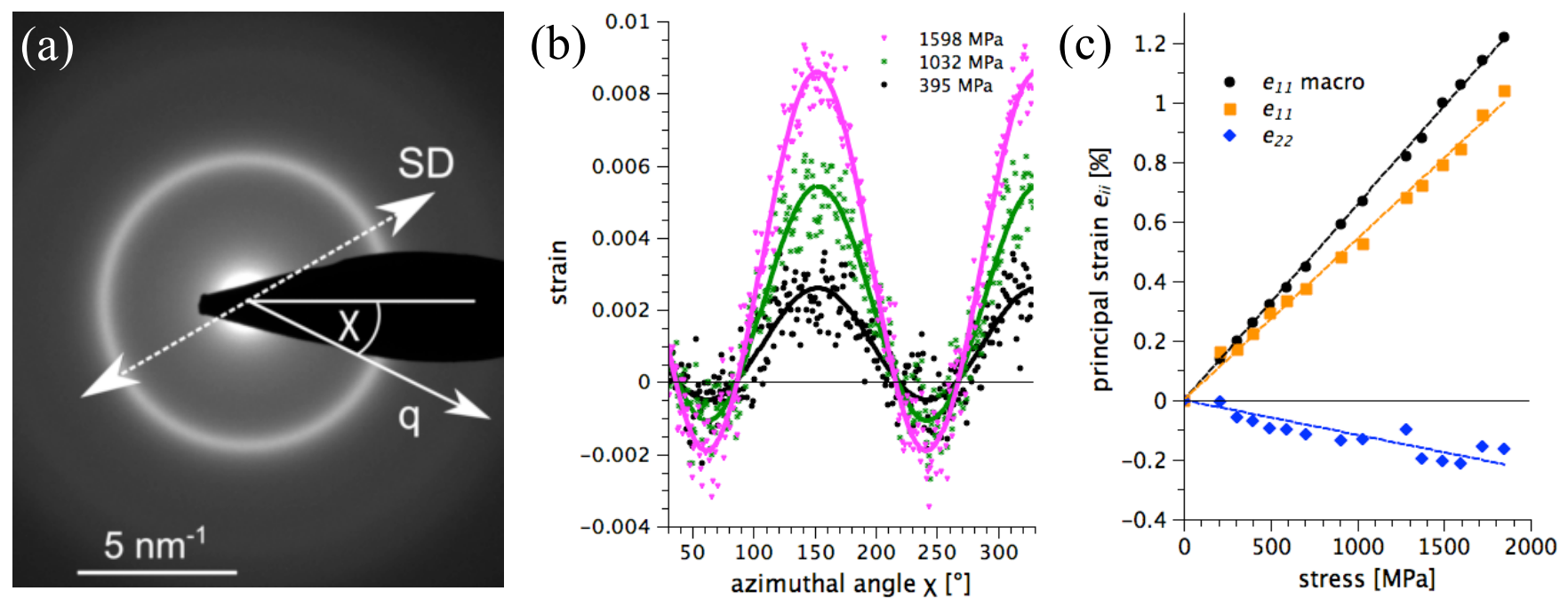

Figure 2. (a) SAED pattern of amorphous TiAl; reciprocal vector $\mathrm{q}$, azimuthal angle $\chi$ and shear direction SD are indicated. (b) Atomic-level elastic strain evaluated from SAED pattern at different stresses. (c) Linear fit of the two principal strains and the macroscopic strain as function of stress.
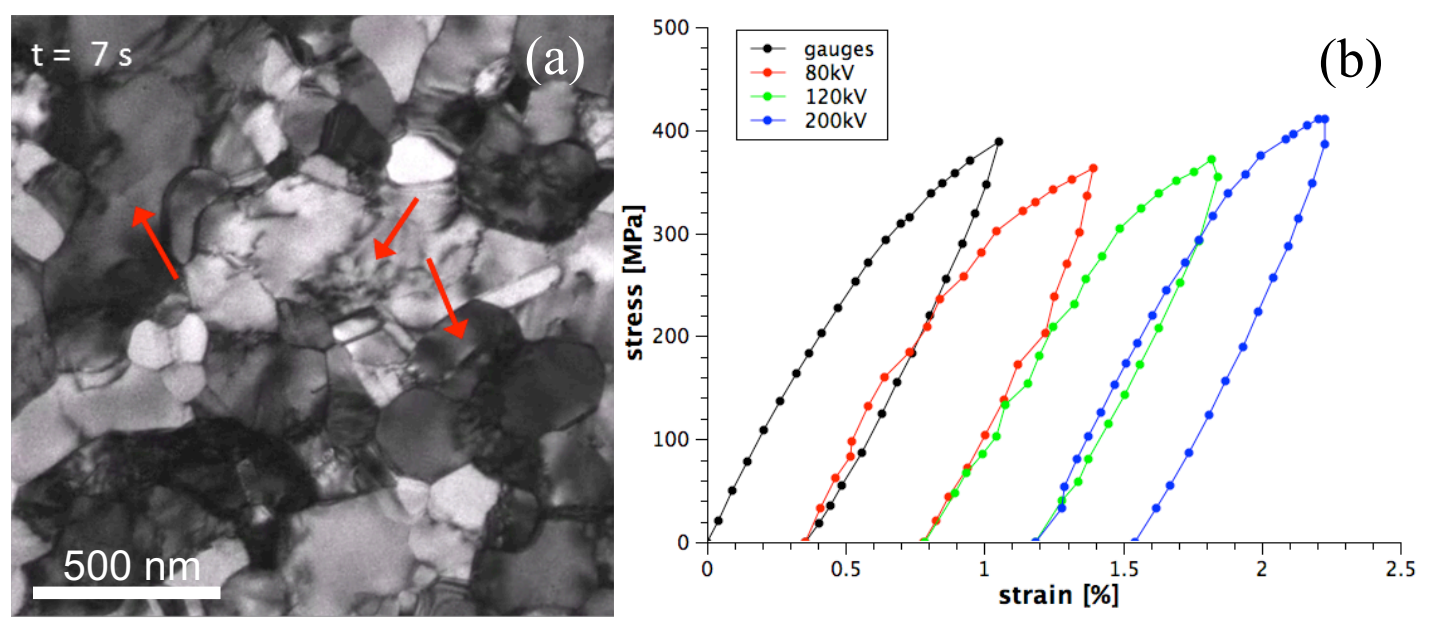

Figure 3. (a) Bright-field image of nanocrystalline Al reveals dislocation activity (red arrows) during insitu straining. (b) Stress-strain cycles obtained at different accelerating voltages indicate e-beam effect. 